\title{
Gastric ulceration as a function of food deprivation in isolated and aggregated mice'
}

WALTER B. ESSMAN

QUEENS COLLEGE OF THE CITY UNIVERSITY OF NEW YORK

The incidence and severity of gastric lesions, developed as a result of $24 \mathrm{hr}$. of food deprivation, was studied in adult mice isolated or aggregated for eight days. Isolated animals showed a reduced amount of food intake, less gastric food retention, a greater incidence of gastric ulcers, and more severe gastric lesions than did the aggregated mice. The isolated animals also showed slightly heavier adrenals and less adrenal ascorbic acid. The data appear consistent with the view that ulcerogenes is in mice is a function of housing conditions which govern activity, food consumption, and the ability to retain food in the stomach.

Gastric ulceration has been experimentally produced in rodents as a result of a variety of agents and/or events. One such event which has been shown to lead to gastric ulcerogenesis in mice is the deprivation of food (Frisone \& Essman, 1965). The extent of the pathology was an inverse function of body weight and the extent to which the retention of gastric food particles occurred. It would appear that, since gastric food retention is related, in one respect, to food consumption, those factors which affect food intake should indirectly affect gastric ulcerogenesis produced by the deprivation of food. In mice, population density and age are determinants of daily food intake. Since the latter variable has been explored and shown to affect the incidence of ulcer development (Frisone \& Essman, 1965) it was the purpose of the present study to consider population density, directed toward a comparison of the effect of post-weaning isolation or aggregation in the genesis of gastric ulceration resulting from food deprivation. It was a further purpose to consider the contribution of isolation or aggregation as possible stressors to ulcerogenesis. Such a distinction was made by a consideration of resulting changes in the adrenals.

Method

Male CF-1 strain mice were selected from litters matched for age and weight. Following group-housing (10 per cage) from weaning (21 days) until 47 days of age the animals were randomly assigned to either isolated $(\mathrm{N}=25)$ or aggregated $(\mathrm{N}=15)$ housing conditions. The isolation conditions consisted of individually housing each animal in a plastic cage $(17 \times 28 \times 13 \mathrm{~cm})$ with ad libitum food and water. The aggregation conditions consisted of housing five animals to an individual cage under the same conditions. ${ }^{2}$ The animals were not handled pre-weaning or post-weaning except at the onset of the experiment ( 47 days of age) and at its conclusion ( 55 days of age).

Daily food consumption was determined by weighing the unconsumed food at $24-\mathrm{hr}$. intervals. Average daily food consumption values were obtained for the grouphoused mice. On the seventh day all food was removed and body weight was recorded for each animal. Following the 24-hr . food-deprivation period the animals were again weighed and then killed with a lethal dose of pentobarbital sodium. The gastric tissue was excised between the oesophagus at the level of the cardiac sphincter and approximately $20 \mathrm{~mm}$ of the duodenum. The tissue was bisected along the greater curvature, the incidence of food particles contained was noted, and following washing it was mounted on index cards. Color positives were made of the gastric tissue and these were rated for the incidence and severity of pathology. The rating scale used was: $0=$ no gastric pathology; $1=$ dilated gastric vessels; 2 =vascular dilation with hemorrhage; 3 = discrete ulceration; and 4 = gross ulceration; with vascular dilation and/or hemorrhage. Several specimens of gastric tissue grossly characterizeä as showing either no pathology or ulceration were fixed, after being photographed, in $10 \%$ formalin for subsequent preparation of histological specimens.

For the ascorbic acid assays the adrenals were removed, weighed, and individually homogenized in 5\% metaphosphoric acid; a $5 \mathrm{ml}$ filtrate was treated with $0.5 \mathrm{ml}$ of 0 -phenylenediamine reagent (0-phenylenediamine, $0.5 \%$ in glycerol, diluted to $10 \%$ in $1 \mathrm{M}$ $\mathrm{NaH}_{2} \mathrm{PO}_{4} \cdot \mathrm{H}_{2} \mathrm{O}$ ). The solutions were agitated and incubated for $1 \mathrm{hr}$. at $32^{\circ} \mathrm{C}$ in the dark. The samples were read against a treated ascorbid acid standard $\left(2 \times 10^{-4} \mathrm{gm} / \mathrm{ml}\right)$ for fluorescence in a Farrand fluorometer using filters for $405 \mathrm{~m} \mu$ peak fluorescence. Results

The histological findings which characterized the gastric tissue which, upon gross inspection, was considered ulcerated, consisted of pathology localized in the body and antral zone of the stomach. There were variations ranging from erosive epithilitis to deeper craters, including infiltration of the muscularis mucosae. There were some instances of necrotic cells and thrombotic inclusions, but no central perforation. Non-ulcerated tissue, in some instances, showed a mild reddening around the fundus and some granulated mucosal tissue.

The results of the comparison between isolated and aggregated animals are shown in Tables 1 and 2 . Whereas there was no statistically significant difference between the animals as far as food consumption over the seven-day feeding period, it may be noted that the aggregated mice did consume somewhat more food. As compared with equivalent initial food intake, measured at the onset of the experiment $(5.30 \mathrm{gm})$, both groups showed a 
Table 1. Food Consumption, Food Retention, and Gastric Ulceration in Isolated and Aggregated Mice

Mean Daily Food PercentGastric PercentGastric Condition $\mathrm{N}$ Consumption(gm) Food Retention Ulceration

\begin{tabular}{|c|c|c|c|c|}
\hline $\begin{array}{l}\text { Aggrejation } \\
\text { (A) }\end{array}$ & 13 & 5.07 & 46 & 46 \\
\hline $\begin{array}{l}\text { Isolation } \\
\text { (1) }\end{array}$ & 25 & 4.54 & 23 & 77 \\
\hline
\end{tabular}

reduction in food consumed by the seventh day. It is of further interest to note that the mean weight loss resulting from the $24-\mathrm{hr}$. period of food deprivation was equal for both groups $(2.8 \mathrm{gm})$.

It can be seen that significantly more of the aggregated mice had undigested food particles remaining in the stomach than did the isolated animals $\left(x^{2}=7.90\right.$; $p<.01)$. The direction of this difference was also consistent for the incidence of gastric ulceration, which was significantly greater for the isolated mice $\left(\chi^{2}=79.10 ; p<.001\right)$, and the rated severity of the gastric pathology, which was also higher for isolated animals $(t=24.00 ; p<.05)$.

Whereas the differences between the adrenal tissue weights $[\Delta(\mathrm{I}-\mathrm{A})]$ and adrenal ascorbic acid concentration $[\Delta(A-I)]$ did not reach acceptable levels of statistical significance, fairly consistent differences obtained when the weights and concentrations were randomly matched between groups. The mean adrenal weight differences and mean adrenal ascorbic acid differences indicated that the isolated mice had heavier adrenals and less ascorbic acid than did the aggregated animals.

\section{Discussion}

The data suggest that the incidence and severity of gastric ulceration occurring in adult mice following $24 \mathrm{hr}$. of food deprivation are related to the predeprivation housing conditions; this relationship appears consistently to suggest that isolation contributed more significantly to ulcerogenesis and gastric pathological severity. Although the observed differences in adrenal weight and adrenal ascorbic acid concentration were not of sufficient magnitude to quantitatively assign either housing condition to a more "stressful" position, the direction of the differences was consistent with predicted adrenal changes resulting from stress; i.e., through randomized matching, those mice with heavier adrenals had less ascorbic acid; such mice were more frequently those which had been isolated.

It seems clear that isolation or aggregation housing for adult mice may serve as a determinant of food

Table 2. Severity of Gastric Lesions and Adrenal Differences as a Function of Isolation and Aggregation

\begin{tabular}{lccc} 
Condition & $\begin{array}{l}\text { Mean Severity of } \\
\left.\text { Gostric Lesion( } \pm_{\sigma}\right)\end{array}$ & $\begin{array}{l}\text { Mean Adrenal } \\
\text { Weight } \Delta(1-A)\end{array}$ & $\begin{array}{c}\text { Mean Adrenal } \\
\text { Ascorbic Acid } \Delta \\
(\text { A-1) }\end{array}$ \\
\hline Aggregation(A) $\quad 1.00 \pm 0.6$ & $00365 \mathrm{mg}$ & $4.08 \mathrm{mM} / \mathrm{kg}$ \\
Isolation(1) & $2.20 \pm 1.2$ & & \\
\hline
\end{tabular}

consumption. Whereas the aggregated animals consumed somewhat more food over the period of the experiment, their weight loss with food deprivation was equivalent to that occurring in isolated animals. There was, however, a more clear relationship between food intake and the retention of gastric food particles; this was significantly more evident for aggregated animals; these animals retained significantly more undigested food particles, showed a significantly lower incidence of ulceration, and a significantly less severe degree of pathology.

It may be concluded, on the basis of the data obtained, that food consumption and food retention is greater for aggregated than for isolated mice and that gastric food retention serves a protection function insofar as ulcerogenesis and ulcer severity are concerned. From the data it appears further that a change from group-housing to isolation in adult mice facilitates ulcerogenesis resulting from $24 \mathrm{hr}$. of food deprivation.

These findings support previous results from this laboratory (Frisone \& Essman, 1965) showing that ulcerogenesis is related to food deprivation and gastric food retention. One parallel between the present findings and ulcerogenesis in rats is the relationship between isolation and aggregation and activity differences. Unpublished data from this laboratory have indicated that with post-weaning isolation mice become more active than do aggregated animals. Rats immobilized during the peak of their activity cycle showed more ulcer development than those immobilized during a trough in the cycle (Ader, 1964). In this same study the author suggested that the presence of gastric food particles was related to the activity differences and that gastric food contents appeared to confer some protection against ulcerogenesis. More recently (Ader, 1965), the same author indicated that activity levels and restraint-induced gastric ulcers differed between isolated and group-housed rats as a function of preweaning handling conditions, but in the absence of handling these differences were not apparent. Our findings are in agreement with the suggestion that gastric ulcerogenesis and its severity occur more frequently in mice which are more active and retain less gastric food; it appears from the data present that these conditions are facilitated by introducing grouphoused adult animals to isolated housing conditions.

\section{References}

Ader, R. Gastric erosions in the rat: effects of immobilization at different points in the activity cycle. Science, 1964, 145, 406407

Ader, R. Effects of early experience and differential housing on behavior and susceptibility to gastric erosions in the rat. $J$. comp. physiol. Psychol., 1965, 60, 233-238.

Frisone, J., \& Essman, W. B. Stress-induced gastric lesions in mice. Psychol. Rep., 1965, 16, 941-946.

\section{Notes}

1. This study was supported in part by USPHS Grant MH-08698-02. 2. Two animals in the aggregated group died on the eighth day of the study. Their gastric and adrenal tissue was not included in the data. 